\title{
MAGNETIC SUSCEPTIBILITY DETERMINATION BASED ON MICROPARTICLES SEDIMENTATION ANALYSIS
}

\author{
Ravnik, J. ; Cernec, D.; Hribersek, M. \& Zadravec, M. \\ University of Maribor, Faculty of Mechanical Engineering, Smetanova 17, SI-2000 Maribor, Slovenia \\ E-Mail: jure.ravnik@um.si, cerdej@yahoo.com,matjaz.hribersek@um.si,matej.zadravec@um.si \\ ( Corresponding author)
}

\begin{abstract}
This paper deals primarily with the sedimentation of magnetic microparticles in the presence of an external magnetic field. Based on the analysis of raster images, taken during sedimentation process under the influence of magnetic field, the development of settling velocities occurring as a result of increasing magnetic force is measured. Basics of the magnetic field calculation of a disc neodymium permanent magnet are explained. Numerical particle tracking and particle size determination via image analysis is presented. On the basis of force balance equations for Lagrangian particle tracking and results of experimental tracking of particle positions during sedimentation with magnetic field turned on as well as off, the magnetic susceptibility of microparticles is determined.

(Received in October 2016, accepted in January 2017. This paper was with the authors 1 month for 1 revision.)
\end{abstract}

Key Words: Sedimentation, Magnetic Microparticles, Magnetic Susceptibility, Image Analysis, Magnetic Field, Magnetic Flux Density

\section{INTRODUCTION}

A significant increase of separation processes using synthesized micro and nanoparticles has provided a lot of new opportunities in recent years, $[1,2]$. Subsequently growth of demands on product properties in this field is also increasing, especially due to large diversity of synthesized particles and their properties, which can vary widely. Recently, synthetization and applications of functionalized magnetic particles has gained much attention, with magnetic particles being coated with various types of coatings [3].

Magnetic separation is a process based on separation of the particles in the presence of a magnetic field, which gives rise to additional forces, acting on the particles [4-6]. In the case of a moving magnetic particle in a non-uniform magnetic field, the Kelvin magnetic force arises. Magnetic susceptibility is an essential property of magnetic particles, and it has a direct influence on the magnitude of the Kelvin magnetic force. Magnetic susceptibility determines the response of a magnetic particle moving through magnetic field and it is one of the essential material properties when designing a magnetic separator unit [7-9]. Since the core of a magnetic particle consists mainly of iron oxides (usually magnetite or hematite), magnetic susceptibility is dependent on mass fraction of iron oxide in the particle. The information on this property along with saturation magnetization of a magnetic particle can sometimes be unknown or inaccurate, so there exists a need to develop feasible procedures for determination of magnetic properties of such particles.

In this article a procedure of magnetic susceptibility determination based on combining sedimentation process and magnetic field effects is described. When a settling magnetic particle is influenced by an external magnetic field, contribution of a magnetic force can be detected as a change of the particle velocity. Procedures for determination of settling velocity are well known, especially in the case of a particle sedimenting under the action of gravity in a fluid at rest [10]. Since particle tracking can be done using camera snapshot settings, the efficiency of performed experiments depends mainly on experimental set-up and quality of the equipment. Additionally, particle can also be tracked numerically. There exist several 
different approaches to particle dynamics modelling, ranging from simple homogenization approaches, suitable for nanoparticles [11, 12], to more complex approaches, taking into account different forces, acting on particles [4, 6, 13]. In our work, the approach based on point particle representation $[1,4]$ was selected.

The paper is structured as follows: experimental part with explanation of settling velocity measurements, magnetic field computation, particle size and centre determination, which is followed by results and conclusions.

\section{EXPERIMENTAL METHOD}

All of the experiments that were performed during the research were essentially observations of sedimentation process. In order to verify proposed measurement method and to determine terminal velocities of magnetic microparticles, preliminary observations and analyses were made without applied magnetic field and therefore experimental work was carried out in three separate stages: experiments with glass spheres, experiments with magnetic particles without the presence of magnetic field and experiments with magnetic particles with the presence of external magnetic field. As the basic setup of all experiments was nearly identical (apart from the absence of the magnet and the way in which glass spheres were dosed) only schematic description of the main experiment (see Fig. 1) is presented. The setup consisted of a transparent glass container with a dosing device for magnetic microparticles at the top of it. On the backside of the container a millimetre scale was placed. The scale was visible on all recordings that were made during the course of experiments and it was essential for later raster image analyses.

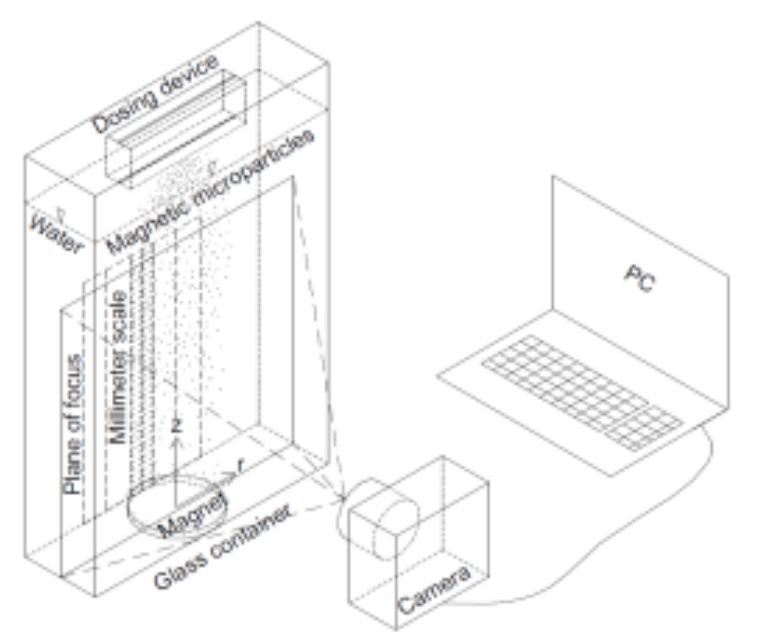

Figure 1: Setup of the main experiment.

Camera-based particle tracking was performed with two types of cameras: Canon Ixus 82IS for the settling of glass spheres and Nikon D5000 for the magnetic particle settling. Before the execution of each experiment water temperature was also measured. Photos i.e. raster images were analysed with an open source image processing program ImageJ.

\section{EXPERIMENTS WITH GLASS SPHERES}

Preliminary analyses were made with glass spheres in the diameter range of 1-1.3 mm. Size of each individual glass sphere was measured manually with digital calliper before it was put into the water at the top of glass container with tweezers. Sedimentation process was recorded with video camera for each individual glass sphere. On the basis of the frame rate of the video recordings, time intervals between successive frames $(0.033 \mathrm{~s})$ were determined. Video 
recordings were then split into separate frames using Video to JPG Converter. As each successive frame was analysed with program ImageJ, the change of a position of a chosen glass sphere in vertical direction was detected. Based on pixel - millimetre ratio vertical distance travelled by glass spheres between successive frames (and thus between known time intervals) were determined and terminal velocities were then calculated. By means of comparison between drag coefficients $\left(C_{d}\right)$ calculated upon balanced forces acting on the glass spheres, and drag coefficients $\left(C_{d R e}\right)$, calculated on the basis of empirical Reynolds number values $(R e)$, verification of previously described measurement method was made. As mentioned above, $C_{d}$ was calculated with the help of measured terminal velocities at which the resistance force, the buoyancy and gravity are balanced:

$$
C_{d}=\frac{2\left(\rho_{d}-\rho_{t}\right) \boldsymbol{g} V_{d}}{\rho_{t} \mathbf{v}_{\mathbf{d}}^{2} A_{d}}
$$

$R e$ was calculated using the expression for a motion of a sphere through a fluid:

$$
R e=\frac{v_{d} \rho_{t} d}{\mu}
$$

Reynolds number values for glass spheres were mainly in the range of $200<R e<1000$ thus the following empirical expression for $C_{d R e}$ was used:

$$
C_{d R e}=\frac{24}{R e}\left(1+0.15 R e^{0.687}\right)
$$

Taking into consideration that glass spheres were put into the water with tweezers and not via dosing device (which was not suitable for glass spheres as they were too big), the comparison between $C_{d}$ and $C_{d R e}$ showed satisfactory results. Ratio $C_{d} / C_{d R e}$ was almost always in the range of $0.6 \div 0.8$, which means that $C_{d R e}$ value was always higher than that of $C_{d}$. $C_{d}$ values varied from 0.5 to 0.63 and $C_{d R e}$ values were between 0.7 and 0.8 . In other words, this comparison showed us, that measured settling velocities were a bit higher than expected. It was established that the main reason for such deviations was the usage of tweezers as a "dosing device" which resulted in some unwanted acceleration at the beginning of the settling process. Nevertheless the results were good enough to verify the measurement method.

\section{EXPERIMENTS WITH MAGNETIC MICROPARTICLES}

When first experiments with magnetic microparticles were performed it became evident, that the highest possible resolution of the recorded videos $(1280 \times 720$ pixels $)$ would not suffice for our purposes as the particles were too small and their shapes were unclear. Therefore it was decided that the camera had to be set to record a continuous series of images. Thus full resolution images $(4288 \times 2848$ pixels $)$ were obtained with the pixel size of approx. one-fifth of the nominal size of the particles. From multiple particles in the images we were now able to pick out spherical ones (as the resolution was still too low to analyse the clusters and nonspherical particles, these were excluded from further analyses). Fig. 2 shows three consecutive images merged into one. In this merged photo the particles are settling in the region of $70 \mathrm{~mm}$ above the surface of magnet (millimetre scale can be also seen in the background).

Three consecutive positions of a single spherical particle (marked with red squares) are also shown, since such small regions were subsequently isolated with a cropping technique and analysed separately to determine size and centre of the particles (as will be later explained in greater detail). 


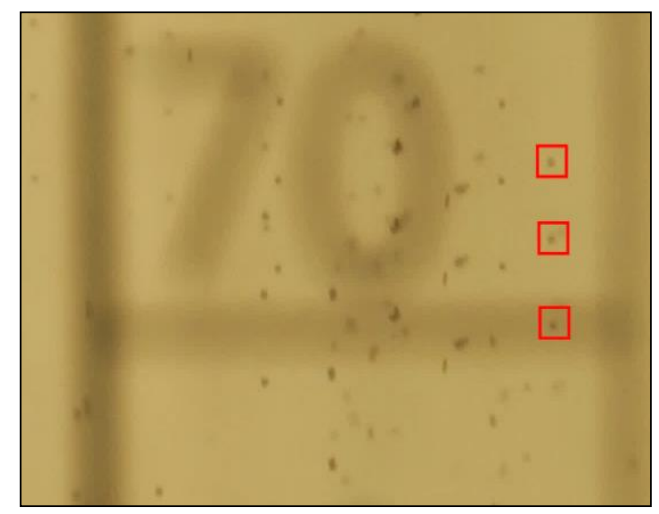

Figure 2: Merged photo of the settling magnetic microparticles.

Disadvantage regarding recordings of continuous series of images was determination of exact time intervals between two consecutive images. Although this data can be found in camera's manual $(0.25 \mathrm{~s})$ it cannot be taken for granted as it depends on the ambient lighting, resolution, focus and other settings on the camera. Therefore a test series of recordings were made with a timer fixed on a front side of the glass container. It was established that the time intervals of $0.28 \mathrm{~s}$ were most frequent, but nevertheless oscillations were still occurring. As a result measured settling velocities were affected by these oscillations and therefore moving average was used to smooth velocity curves.

\subsection{Settling without magnetic field influence}

As mentioned before, our main focus during research was to analyse the behaviour of the settling magnetic microparticles in applied external magnetic field. However, in order to exclude unwanted factors and determine terminal velocity values of magnetic microparticles, at first analyses without applied magnetic field were made. At the beginning our idea was to determine drag coefficient values for particles of the same sizes at different settling velocities i.e. at different water temperatures. It was our purpose to use these values for later calculations at higher settling velocities when magnetic particles would move in the presence of magnetic field, but this idea was later abandoned as intensified water flows at higher water temperatures $\left(30^{\circ} \mathrm{C}\right.$ and more) were becoming a dominant factor. It also became obvious that the sizes of the particles were too diverse (from 70 to $150 \mu \mathrm{m}$ ) to accomplish such a task. However, analyses that were made without magnetic field influence, did prove useful for further experimental work as some terminal velocities of different sized particles were determined at lower temperatures. At this stage of research we noticed some similarities to the sedimentation process of the glass spheres as $C_{d}$ values also differed from that of $C_{d R e}$ (Table I), although at this time not for the same reason, as in contrast to the glass spheres, magnetic microparticles entered the water via dosing device without unwanted accelerations at the start of the settling process.

Table I: Comparison between $C_{d}$ and $C_{d R e}$ for magnetic microparticles - settling at $24.1^{\circ} \mathrm{C}$.

\begin{tabular}{|c|c|c|c|}
\hline $\begin{array}{c}\text { Magnetic } \\
\text { particle }\end{array}$ & $\begin{array}{c}\text { Diameter } \\
(\mathrm{mm})\end{array}$ & $\boldsymbol{C}_{\boldsymbol{d}}$ & $\boldsymbol{C}_{\boldsymbol{d R} \boldsymbol{e}}$ \\
\hline $24-6-2$ & 0.133 & 31.314 & 42.249 \\
\hline $24-6-3$ & 0.129 & 30.504 & 43.609 \\
\hline $24-6-4$ & 0.149 & 43.276 & 42.034 \\
\hline $24-6-5$ & 0.133 & 36.579 & 45.540 \\
\hline $24-6-6$ & 0.147 & 35.886 & 39.097 \\
\hline $24-6-7$ & 0.142 & 55.968 & 50.722 \\
\hline $24-6-8$ & 0.135 & 65.215 & 8.649 \\
\hline
\end{tabular}


Due to much lower settling velocities $\left(\boldsymbol{v}_{k p}\right)$ of magnetic particles $(3-4 \mathrm{~mm} / \mathrm{s}$ in comparison to the velocities of glass spheres which varied between 170 and $210 \mathrm{~mm} / \mathrm{s}$ ) we assumed that these differences could have been caused by local water flows. $C_{d R e}$ values were not always higher compared to these of $C_{d}$ (as in the case of glass spheres) and therefore our conclusion was that for $C_{d}<C_{d R e}$ local flow contributes to the settling velocity and for $C_{d}>C_{d R e}$ it reduces this velocity. Local flow velocities $\left(\boldsymbol{v}_{t v}\right)$ were estimated upon determination of the settling velocities at which $C_{d}$ and $C_{d R e}$ coincide $\left(C_{d} \approx C_{d R e}\right)$. Based on equation:

$$
\boldsymbol{v}_{k p}=\boldsymbol{v}_{k p_{-} k} \pm \boldsymbol{v}_{t v}
$$

corrected terminal velocities $\left(\boldsymbol{v}_{k p_{-} k}\right)$ were then calculated.

Reynolds number values at terminal velocities of magnetic microparticles were in the range of $0.2>R e>2$ and $C_{d R e}$ was determined upon empirical expression:

$$
C_{d R e}=\frac{24}{R e}\left(1+0.1 R e^{0.99}\right)
$$

As Reynolds number values were quite low, it was also reasonable to make comparison to Stokes settling velocity $\left(\mathbf{v}_{k p_{-} s t}\right)$, given by expression:

$$
\mathbf{v}_{k p_{-} S t}=\frac{d^{2}\left(\rho_{d}-\rho_{t}\right) \boldsymbol{g}}{18 \mu}
$$

which predicts the settling velocity of small spheres in fluid. The results can be seen in Table II.

Table II: Comparison between settling velocities at $24.1^{\circ} \mathrm{C}$.

\begin{tabular}{|c|c|c|c|}
\hline $\begin{array}{c}\text { Magnetic } \\
\text { particle }\end{array}$ & $\begin{array}{c}\mathbf{v}_{k p} \\
(\mathrm{~mm} / \mathrm{s})\end{array}$ & $\begin{array}{c}\mathbf{v}_{k p_{-} k}(\mathrm{~mm} / \mathrm{s}) \\
\left(C_{d} \approx C_{d R e}\right)\end{array}$ & $\mathbf{v}_{k p_{-} s t}(\mathrm{~mm} / \mathrm{s})$ \\
\hline $24-6-2$ & 4.114 & 3.096 & 3.235 \\
\hline $24-6-3$ & 4.104 & 2.946 & 3.040 \\
\hline $24-6-4$ & 3.700 & 3.795 & 4.043 \\
\hline $24-6-5$ & 3.804 & 3.090 & 3.228 \\
\hline $24-6-6$ & 4.041 & 3.715 & 3.954 \\
\hline $24-6-7$ & 3.179 & 3.490 & 3.685 \\
\hline $24-6-8$ & 2.872 & 3.175 & 3.332 \\
\hline
\end{tabular}

It is evident, that as soon as local water flows are taken into account, the new, corrected settling velocities almost match Stokes settling velocities, the only reason for slight differences being relatively high $R e$, as Eq. (5) is supposed to be used for $R e \ll 1$. But as continuation of our experimental work focused on the sedimentation process in the presence of the magnetic field, where particle velocities were eventually much higher than initial settling velocities (i.e. terminal velocities), this difference was neglected and expression for Stokes settling velocity was used to estimate terminal velocities, as (with magnetic force involved) the measurement of these velocities would have been questionable even at greater distances from the magnet.

\subsection{Settling in the presence of magnetic field}

Basic experiment setup for particle tracking in the presence of magnetic field (see Fig. 1) along with recording procedure was similar to those of previously performed experiments, although adjustments of the camera's position were a bit trickier at this time, since the optimal region for observations was limited through magnetic field characteristics. Namely, as magnetic particles were approaching the magnet at the bottom of the glass container it was 
possible to see, even with the naked eye, that in the close neighbourhood of the magnet $(\sim 30$ $\mathrm{mm}$ and less above its surface), magnetic particles were no longer moving strictly in the vertical direction and at the same time the velocities seemed much higher in this region. On the basis of these observations and computation of the magnetic field the optimal region for particle tracking was determined as shown in Fig. 3.

As shown, tracking of the particle trajectories was recorded around a magnet's axis (from $r=0$ to $\sim 15 \mathrm{~mm}$ ) at a height of $\sim 35$ to $\sim 75 \mathrm{~mm}$ above the magnet's surface. In this region tracking of the particles was well controlled, since the movement in a radial direction was not present and the particle velocities were still relatively low. Magnetic field around the disc magnet and its characteristics in the region, where particle tracking was performed, is explained in the next chapter.

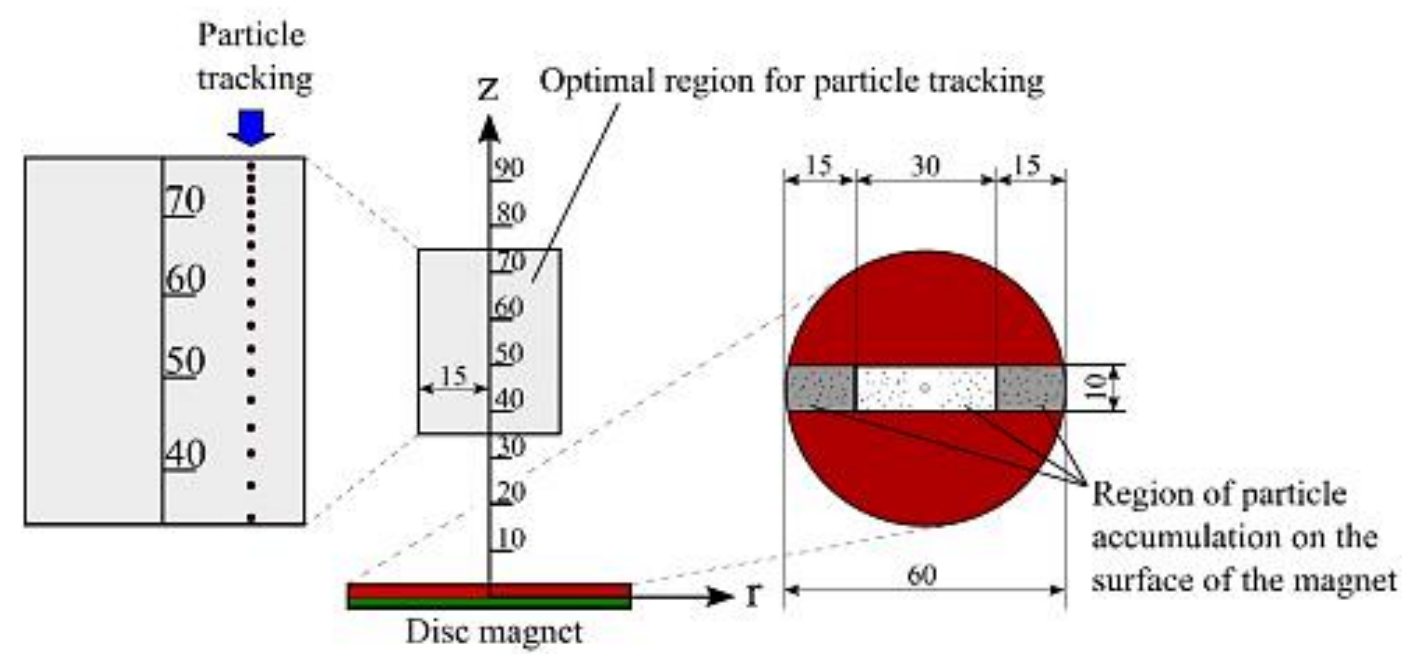

Figure 3: Optimal region for particle tracking.

\section{MAGNETIC FIELD COMPUTATION}

As a source of magnetism, axially magnetized permanent neodymium disc magnet $(60 \mathrm{~mm}$ diameter $\times 5 \mathrm{~mm}$ thick) with magnetization grade N42 was used. Size and shape of the magnet were quite suitable for lowering the magnet (with help of an aluminium holder) into the water and with some adjustments it was properly positioned at the bottom of the glass container (see Fig. 4).

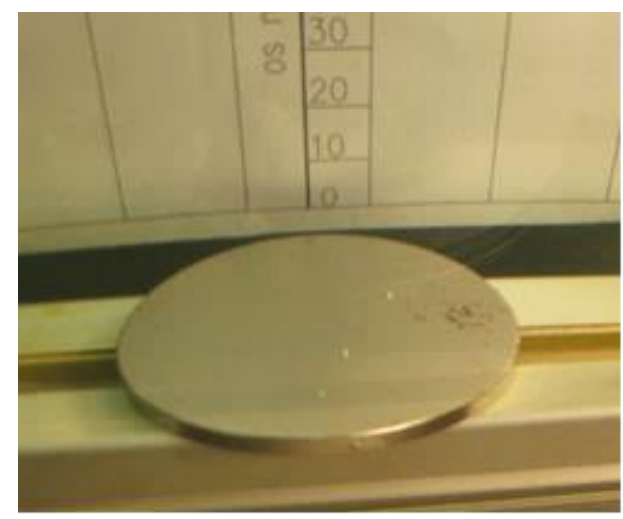

Figure 4: Magnet's position at the bottom of the glass container.

In general an axially magnetized disc-shaped magnet generates axisymmetric magnetic field and along with known magnetization grade enough information is given to compute the magnetic field. A simple illustration of magnetic field around the chosen magnet can be seen 
in Fig. 5, where cylindrical coordinate system and characteristic section of the magnet are also introduced.

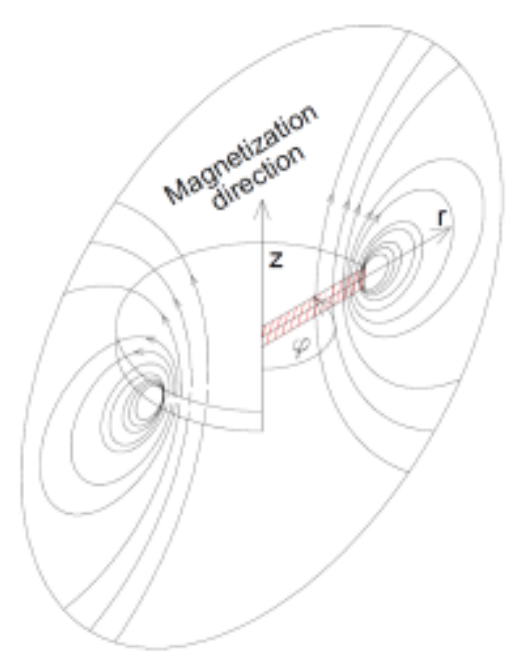

Figure 5: Magnetic field around the chosen disc-shaped magnet.

The force on magnetic particle moving through an applied magnetic field is given by the expression:

$$
\boldsymbol{F}_{\text {mag }}=\frac{\Delta \chi \cdot V_{d}}{\mu_{0}} \cdot(\nabla \boldsymbol{B}) \cdot \boldsymbol{B}
$$

where $V_{d}$ is the volume of the particle, $\Delta \chi$ is the difference in susceptibility between the particle and the fluid (water, in this specific case) and $\mu_{0}$ the magnetic permeability of free space. In order to determine magnetic force $\left(\boldsymbol{F}_{\text {mag }}\right)$, magnitude of magnetic flux density $(\boldsymbol{B})$ and its gradient $(\nabla \boldsymbol{B})$ are also needed and can only be obtained when magnetic field characteristics are known for any position of the magnetic particle moving (i.e. settling) through this field.

The magnetic field was computed with finite element package (Finite Element Method Magnetics: FEMM 4.2, [14]) for solving 2D planar and axisymmetric problems. Initial settings were made for solving axisymmetric problem in the cylindrical coordinate system. With the origin of the coordinate system positioned at the centre of the magnet, characteristic section of the magnet was defined as shown in Fig. 6.

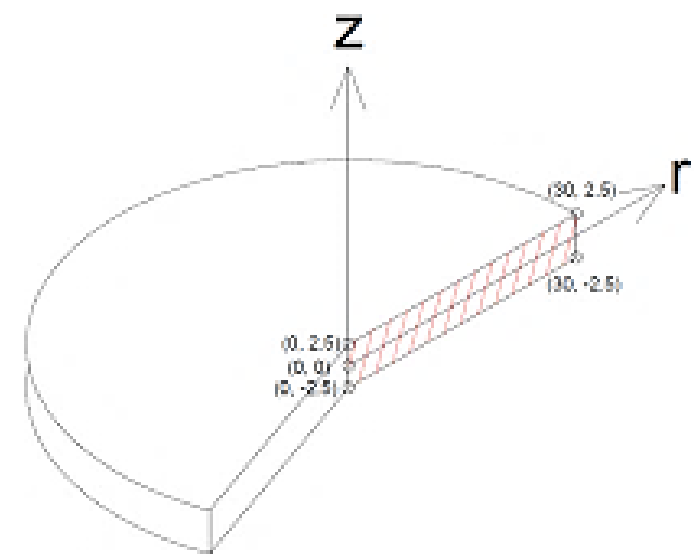

Figure 6: Characteristic section of the magnet in cylindrical coordinate system.

Because axisymmetric nature of the problem means that characteristics of the magnetic field are independent of angular coordinate $\varphi$, the computation of the magnetic field for characteristic section of the magnet gives us all information needed for further analyses. 
Although in the present article FEMM 4.2 and all of its functions cannot be explained in greater detail (anyway, extensive manual and examples can be found on the Internet), there are a few things that must also be mentioned with reference to the subject mentioned above. After definition of the characteristic section of the magnet, boundary conditions must also be given (in this specific case it was circular region with radius of $70 \mathrm{~mm}$ ) and in addition to that, if "Open Boundary Builder" option is selected, the "infinite" magnetic field computation can be performed, meaning that magnetic field does not completely vanish at $70 \mathrm{~mm}$ from the origin (centre of the magnet), but it also spreads beyond this boundary (corresponding to the actual magnetic field around the magnet). Though the results of the magnetic field simulation (see Fig. 7) show that magnetic field is relatively weak at $\boldsymbol{z}>70 \mathrm{~mm}$, having available information for outer region too was very useful, as the region for particle tracking was not strictly limited to $\boldsymbol{Z}<70 \mathrm{~mm}$.

Since no scale can be seen in Fig. 7 (it is just a colour presentation of the magnetic flux density distribution without particular accuracy) the value of the $\boldsymbol{z}$ coordinate can be estimated by length $(30 \mathrm{~mm})$ and height $(5 \mathrm{~mm})$ of the characteristic section of the magnet. Still it is evident that the conditions around magnet axis $(\boldsymbol{r}=0$ to $\sim 15 \mathrm{~mm})$ and at higher $\boldsymbol{Z}$ values (35 mm and more) are much less intense (light blue) as these at the poles of the magnet (yellow and purple). This finding corresponds with observations of the magnetic particle movement and thus with the optimal region for particle tracking (see Fig. 3).
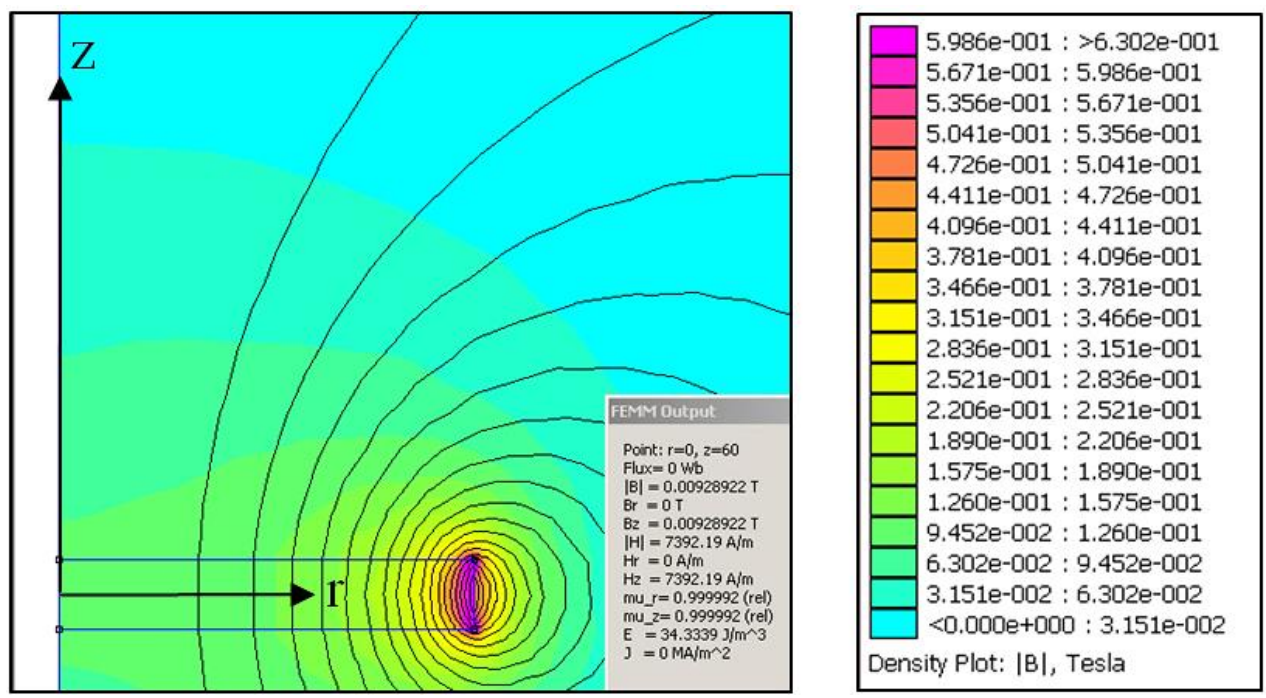

Figure 7: Colour presentation of the magnetic field for characteristic section of the magnet.

As there is no option for magnetic flux density gradient $\nabla \boldsymbol{B}$ calculation in FEMM 4.2 postprocessor window, a central difference method was used to calculate these gradients upon the magnetic flux density $\boldsymbol{B}$ data (for this purpose Lua-script was written and executed through "Lua console window" - an option available in FEMM 4.2 post-processor window). Afterwards all data was exported in .DAT file and then converted into Excel, where the process was automatized to calculate $\boldsymbol{B}, \nabla \boldsymbol{B}$ and derivatives for any position of the particle in the magnetic field. It must also be mentioned, that prior to the execution of the magnetic field computation some adjustments of mesh density can prove useful, since the default settings with low mesh density can be unsuitable for later gradient calculations.

For the purpose of the particle tracking only data in the range from $r=0-20 \mathrm{~mm}$ and $z=10-90 \mathrm{~mm}$ (a little bit wider region in comparison to optimal region for particle tracking) in steps of $0.1 \mathrm{~mm}$ was exported (as can be seen in Fig. 8, where $T_{p}$ marks the position of the particle surrounded with its neighboring coordinates, which are needed to calculate field gradient by means of the central difference method). 


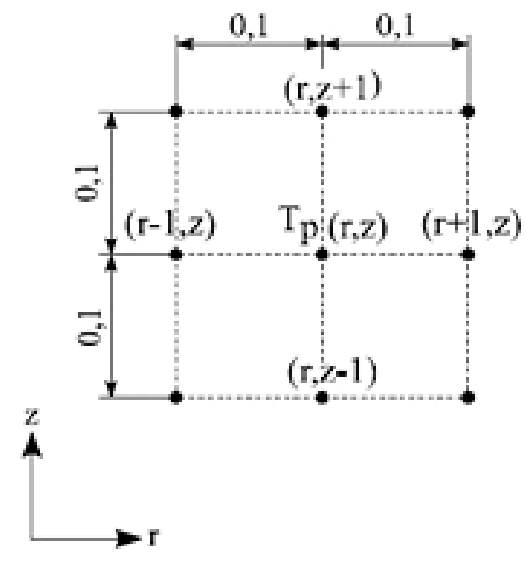

Figure 8: Coordinates of the settling particle $(r, z)$ and neighbouring coordinates.

\section{MAGNETIC PARTICLE SIZE AND CENTER DETERMINATION}

Particle size determination was based on raster image analysis. In order to isolate single particles, outer parts of the source images were removed. The source images were cropped in such a way that, as a result, the final images consisted solely of a darker area (particle) and its brighter surroundings (water). All images of these isolated particles (as shown in Fig. 9) were then subject to analysis in Mathematica. For this purpose code was written to evaluate degrees of darkness of the darker area, using standard deviation as an indicator (the guideline being declining darkness intensity from the centre of the particle to its outside edge).

Calibration of the evaluation process was made upon comparison between measured particle velocities (particle settling without applied magnetic field) and Stokes settling velocities. Settling velocity of a selected particle was measured as described in section 2 and the diameter was determined in ImageJ via pixel - millimetre ratio. Furthermore the value of the measured diameter was being adjusted until both velocities were equal and thus real particle size was determined. In this way more images were examined and afterwards each of them was analysed in Mathematica to determine a deviation factor $f$. It was established that the value of $f$ fell within the region of one and two standard deviations ( $\sigma$ and $2 \sigma$ ) of the peak value (center of the particle). Upon the observations and comparisons the decision was made that $f=1.125$ would be used for particle size determination. As already pointed out, the peak value i.e. darkest spot in the image also represented the centre of the particle (marked with a small red circle in Fig. 9).

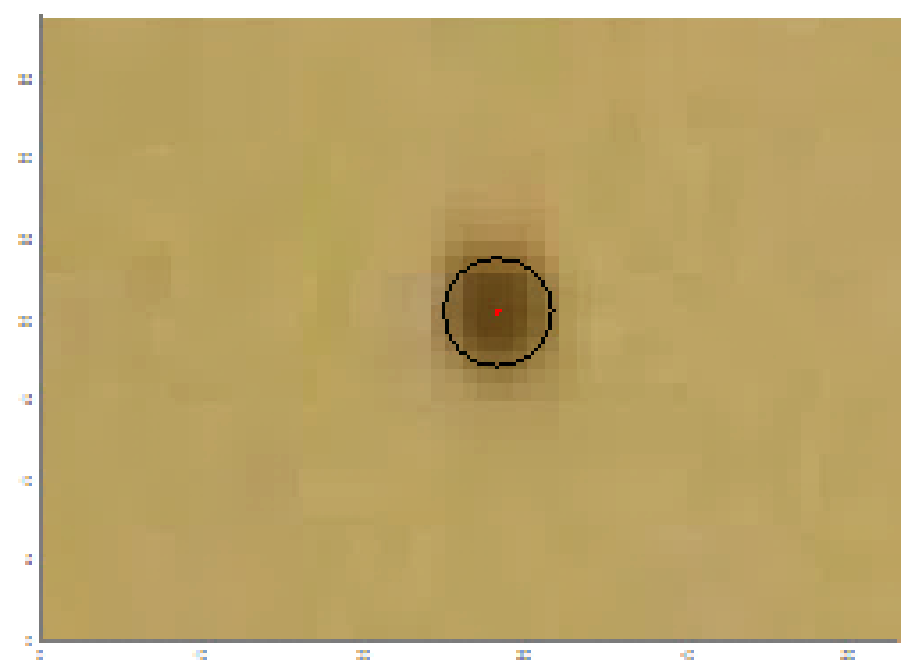

Figure 9: Determination of particle size (bigger black circle) and centre (smaller red circle). 
Gaussian distribution of the intensity of the darker pixels, representing a particle, is shown in Fig. 10. For each settling magnetic particle at least 21 images were analysed in accordance with the procedure described above and obviously particle size values varied from image to image. Therefore the mean value of the particle diameter was used for calculations of the magnetic force.

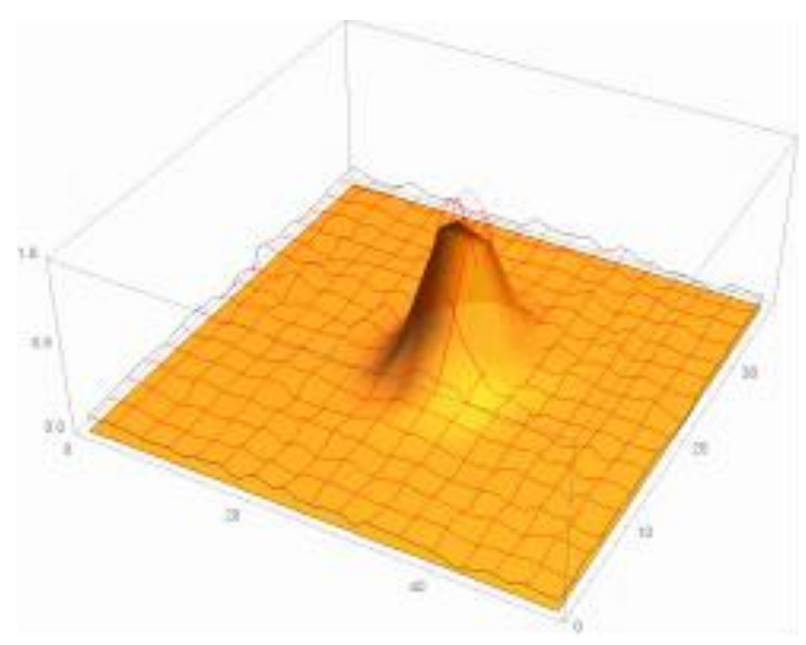

Figure 10: Gaussian distribution of the intensity of the darker pixels.

\section{MAGNETIC FORCE, MAGNETIC SUSCEPTIBILITY AND RESULTS}

The experimental results of particle sedimentation can be effectively used in combination with a numerical model, describing translational momentum conservation, i.e. particle acceleration due to the action of different forces on a particle, to derive a model for determination of particle properties.

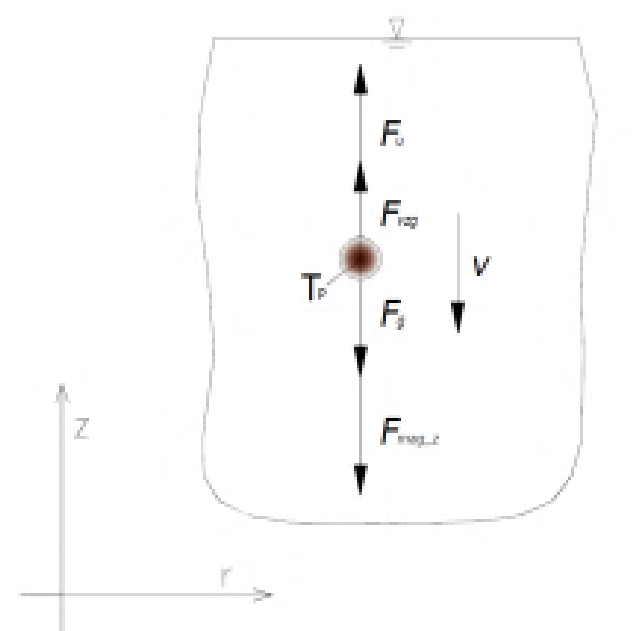

Figure 11: Gaussian distribution of the intensity of the darker pixels.

As can be seen in Fig. 11, showing forces on a settling magnetic microparticle in the presence of the magnetic field, the drag force $\boldsymbol{F}_{u}$, buoyancy $\boldsymbol{F}_{v z g}$ and gravity $\boldsymbol{F}_{g}$ as well as the magnetic force $\boldsymbol{F}_{\text {mag_z }}$ (vertical component of magnetic force) are acting on the particle. The inertia force $\boldsymbol{F}_{\text {net }}$ can therefore be expressed as:

$$
\boldsymbol{F}_{\text {net }}=\boldsymbol{F}_{\text {mag } z}+\boldsymbol{F}_{g}-\boldsymbol{F}_{\text {vzg }}-\boldsymbol{F}_{u}
$$

and thus differential equation for settling of a particle in the presence of a magnetic field can be written: 


$$
m \frac{d \boldsymbol{v}}{d t}=\boldsymbol{F}_{m a g_{-} z}+\left(\rho_{d}-\rho_{t}\right) \boldsymbol{g}\left(\rho_{d}-\rho_{t}\right) \boldsymbol{g} V_{d}-C_{d} \rho_{t} \frac{\boldsymbol{v}_{\boldsymbol{d}}^{2}}{2} A_{d}
$$

where $\boldsymbol{F}_{\text {mag_z }}$ is unknown. However, now that magnetic field characteristics (i.e. magnetic flux density along the trajectory of a settling particle) are being defined upon magnetic field computation, Eq. (7) can be implemented to solve this problem. Since the problem is independent of angular coordinate $\varphi$ (a characteristic of the axisymmetric magnetic field), Eq. (7) can be written as:

$$
\boldsymbol{F}_{m a g}=\frac{\Delta \chi V_{d}}{\mu_{0}} \cdot\left(\begin{array}{l}
\boldsymbol{B}_{r} \frac{\partial \boldsymbol{B}_{r}}{\partial r}+\boldsymbol{B}_{z} \frac{\partial \boldsymbol{B}_{r}}{\partial z} \\
\boldsymbol{B}_{r} \frac{\partial \boldsymbol{B}_{z}}{\partial r}+\boldsymbol{B}_{z} \frac{\partial \boldsymbol{B}_{z}}{\partial z}
\end{array}\right)
$$

where $(\nabla \boldsymbol{B}) \cdot \boldsymbol{B}$ part of the magnetic force is reduced to components $r$ and $z$ (expressed in brackets) and finally $\boldsymbol{F}_{\text {mag_z }_{-}}$can be given by the expression:

$$
\boldsymbol{F}_{\text {mag_z }}=\frac{\Delta \chi V_{d}}{\mu_{0}} \cdot\left(\boldsymbol{B}_{r} \frac{\partial \boldsymbol{B}_{z}}{\partial r}+\boldsymbol{B}_{z} \frac{\partial \boldsymbol{B}_{z}}{\partial z}\right)
$$

Expression in brackets in the Eq. (11) is also called magnetic force density (hereafter referred to as $((\nabla \boldsymbol{B}) \cdot \boldsymbol{B})_{z}$ and the only unknown left in this equation is $\Delta \chi$ (as a matter of fact, just magnetic susceptibility of magnetic particle $\chi_{d}$ is unknown, since magnetic susceptibility of water is known to be $\chi_{v}=-0.000009035$; negative value resulting from diamagnetic nature of water).

By combining Eqs. (9) and (11) we can now express $\boldsymbol{F}_{\text {mag_z }}$ and write:

$$
\boldsymbol{F}_{\text {mag_z }_{-}}=\frac{\Delta \chi V_{d}}{\mu_{0}} \cdot((\nabla \boldsymbol{B}) \cdot \boldsymbol{B})_{z}=m \frac{d v}{d t}+C_{d} \rho_{t} \frac{v_{d}^{2}}{2} A_{d}-\left(\rho_{d}-\rho_{t}\right) \boldsymbol{g} V_{d}
$$

where $((\nabla \boldsymbol{B}) \cdot \boldsymbol{B})_{z}$ is calculated with reference to particle position in the magnetic field and velocity (together with its first time-derivative) is determined upon particle tracking and image analyses.

Rearranging Eq. (12) we can isolate $\Delta \chi$ :

$$
\Delta \chi=\left(m \frac{d \boldsymbol{v}}{d t}+C_{d} \rho_{t} \frac{\boldsymbol{v}_{\boldsymbol{d}}^{2}}{2} A_{d}-\left(\rho_{d}-\rho_{t}\right) \boldsymbol{g} V_{d}\right) \cdot\left(\frac{\mu_{0}}{V_{d}((\nabla \boldsymbol{B}) \cdot \boldsymbol{B})_{z}}\right)
$$

and finally upon:

$$
\chi_{d}=\Delta \chi+\chi_{v}
$$

magnetic susceptibility of a magnetic particle can be calculated.

In the present study, the magnetic susceptibility was calculated for 30 particles, moving through the optimal region (Fig. 3). At least 21 positions i.e. images per particle were analysed (altogether 645 images). In the optimal region, where recordings were made, saturation magnetization $\left(\boldsymbol{M}_{s a t}\right)$ of magnetic microparticles is not yet achieved and therefore magnetic susceptibility for the chosen particle is not limited to just one value. As shown in Fig. 12, magnetic susceptibility $\chi_{d}$ decreases with increase in magnetic flux density $\boldsymbol{B}$, since the distance between particle and magnet reduces with each consecutive image. This behaviour corresponds with definition of magnetic susceptibility:

$$
\chi=\frac{M}{\boldsymbol{H}}
$$

where $\boldsymbol{M}$ stands for magnetization and $\boldsymbol{H}$ for magnetic field strength, which are both closely related to magnetic flux density $\boldsymbol{B}$ : 


$$
\boldsymbol{B}=\mu_{0}(\boldsymbol{H}+\boldsymbol{M})
$$

As for $\chi_{d}$ in Fig. 12 it is also worth mentioning that these values represent just a part of a complete $\chi_{d}-\boldsymbol{B}$ curve, since the optimal region, where tracking of the particles was performed, lies in low magnetic field intensity.

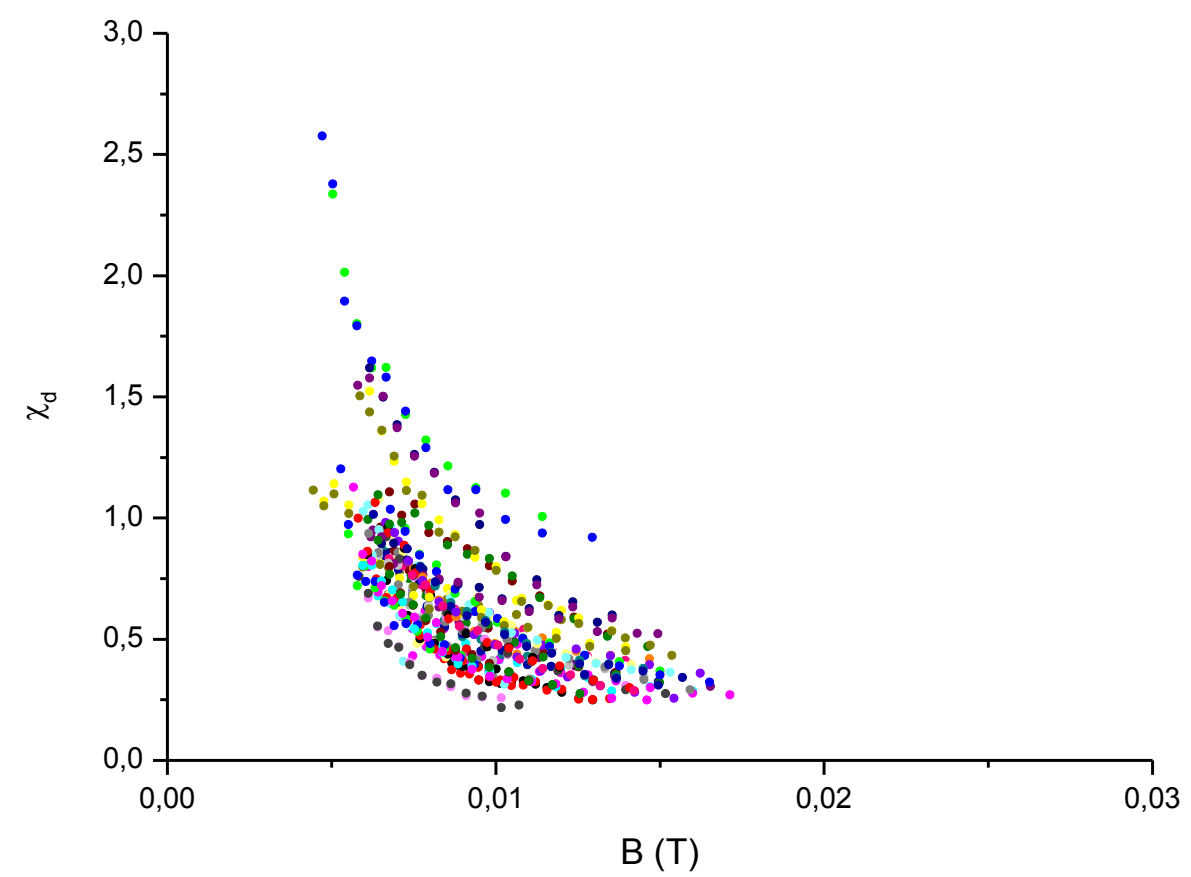

Figure 12: Magnetic susceptibility of magnetic microparticles $\chi_{\mathrm{d}}$ in relation to magnetic flux density B.

To get a better understanding of the situation, a plot of the complete $\chi_{d}-\boldsymbol{B}$ curve was also made (Fig. 13). This simulation was performed on the basis of a simulation model originating from similar research [4]. As can be seen, results of the experiments lie in the upper part of the simulated curve and thus still far from saturation.

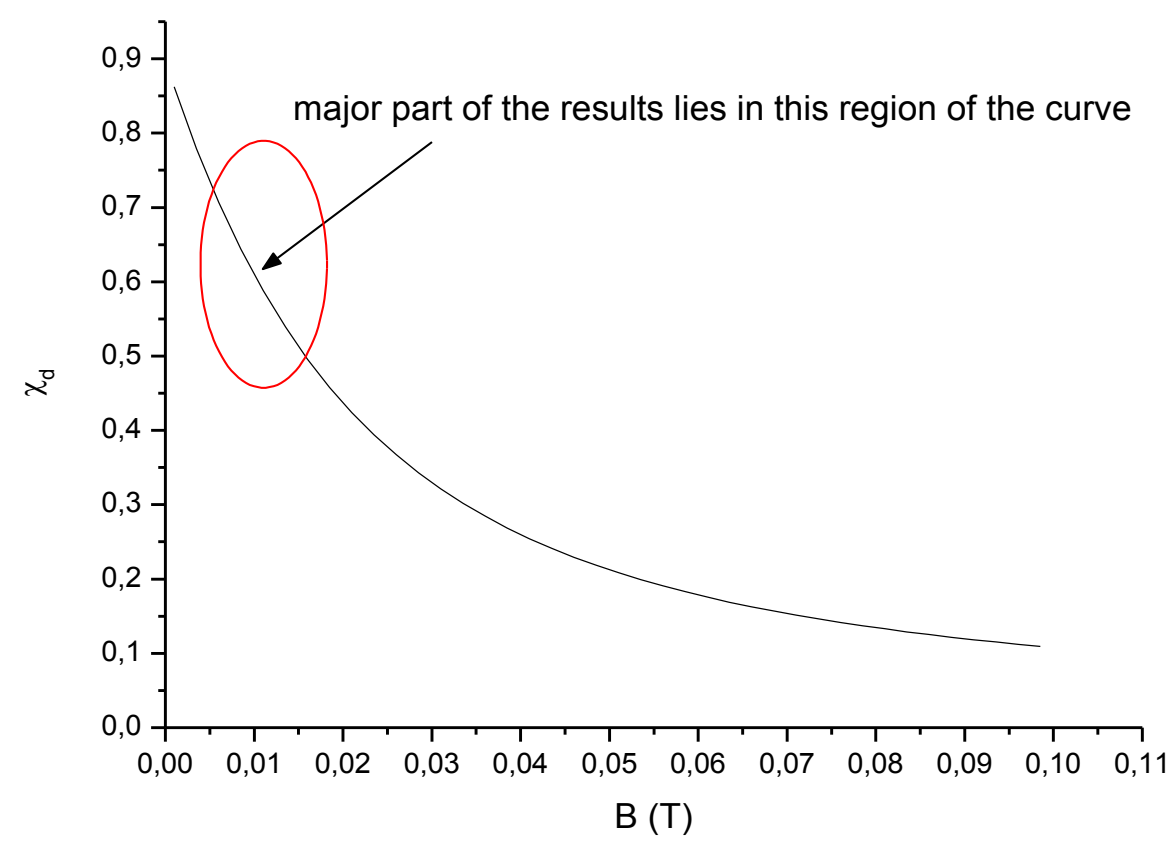

Figure 13: Position of the major part of the results on a complete $\chi \mathrm{d}-\mathrm{B}$ curve. 


\section{CONCLUSIONS}

The main goal of the presented study was to show eventual new possibilities of experimental work in the field of particle sedimentation with magnetic particles being involved in the process. It was established that settling velocities and diameters of magnetic microparticles can be obtained through 2D image analyses, although with some restrictions. We were facing the main difficulty in the resolution of the images. Since the resolution was too low to analyse clusters and non-spherical shapes, only spherical particles were analysed. Additionally, local convective flows were causing some problems as well as evidentially it was impossible to keep the water perfectly still and subsequently particle velocities were subject to corrections. Obviously, for similar future experimental work this can mainly be avoided by some experimental set-up improvements, better camera and additional equipment. Taking into account, that enough initial information is given for a chosen magnet, magnetic field computation can be performed with relative ease if fundamental models of FEMM 4.2 package are mastered. However, due to limitations of 2D image analysis, optimal region for particle tracking must be carefully chosen for the represented method to work properly, since particle position in the direction of $r$-axis can only be controlled in the plane of focus and therefore low intensity magnetic field region must be determined with magnetic force dominating in the direction of $z$-axis.

Finally it must also be mentioned that the comparison between magnetic susceptibilities originating from particle-manufacturer data and susceptibilities resulting from presented experimental work was also made. It was established that experimental values were higher (mainly in the range from 0.4 to 0.8 ) than manufacturer-based values (from 0.09 to 0.13 ). But with some caution we can say that manufacturer data is at least a bit dubious, as to get such magnetic susceptibility values, the settling velocities should have been lower than Stokes settling velocities and that can obviously not be true.

\section{REFERENCES}

[1] Hriberšek, M.; Žajdela, B.; Hribernik, A.; Zadravec, M. (2011). Experimental and numerical investigations of sedimentation of porous wastewater sludge flocs, Water Research, Vol. 45, No. 4, 1729-1735, doi:10.1016/j.watres.2010.11.019

[2] Lenshof, A.; Laurell, T. (2010). Continuous separation of cells and particles in microfluidic systems, Chemical Society Reviews, Vol. 39, No. 3, 1203-1217, doi:10.1039/b915999c

[3] Eichholz, C.; Stolarski, M.; Goertz, V.; Nirschl, H. (2008). Magnetic field enhanced cake filtration of superparamagnetic PVAc-particles, Chemical Engineering Science, Vol. 63, No. 12, 3193-3200, doi:10.1016/j.ces.2008.03.034

[4] Zadravec, M.; Hriberšek, M.; Steinmann, P.; Ravnik, J. (2014). High gradient magnetic particle separation in a channel with bifurcations, Engineering Analysis with Boundary Elements, Vol. 49, 22-30, doi:10.1016/j.enganabound.2014.04.012

[5] Mahmoud, A.; Olivier, J.; Vaxelaire, J.; Hoadley, A. F. A. (2010). Electrical field: A historical review of its application and contributions in wastewater sludge dewatering, Water Research, Vol. 44, No. 8, 2381-2407, doi:10.1016/j.watres.2010.01.033

[6] Mariani, G.; Fabbri, M.; Negrini, F.; Ribani, P. L. (2010). High-Gradient Magnetic Separation of pollutant from wastewaters using permanent magnets, Separation and Purification Technology, Vol. 72, No. 2, 147-155, doi:10.1016/j.seppur.2010.01.017

[7] Pamme, N. (2006). Magnetism and microfluidics, Lab on a Chip, Vol. 6, No. 1, 24-38, doi: $10.1039 / \mathrm{b} 513005 \mathrm{k}$

[8] Xia, N.; Hunt, T. P.; Mayers, B. T.; Alsberg, E.; Whitesides, G. M.; Westervelt, R. M.; Ingber, D. E. (2006). Combined microfluidic-micromagnetic separation of living cells in continuous flow, Biomedical Microdevices, Vol. 8, No. 4, 299-308, doi:10.1007/s10544-006-0033-0 
[9] Kurt, M.; Duru, N.; Canbay, M. M.; Duru, H. T. (2016). Prediction of magnetic susceptibility class of soil using decision trees, Technical Gazette, Vol. 23, No. 1, 83-90, doi:10.17559/TV20140807111130

[10] Gosar, L.; Steinman, F.; Širok, B.; Bajcar, T. (2009). Phenomenological sedimentation model for an industrial circular settling tank, Strojniski vestnik - Journal of Mechanical Engineering, Vol. 55, No. 5, 319-326

[11] Hosseinzadeh, F.; Sarhaddi, F.; Mohebbi-Kalhori, D. (2016). Numerical investigation of the nanoparticle volume fraction effect on the flow, heat transfer, and entropy generation of the $\mathrm{Fe}_{3} \mathrm{O}_{4}$ ferrofluid under a non-uniform magnetic field, Strojniski vestnik - Journal of Mechanical Engineering, Vol. 62, No. 9, 521-533, doi:10.5545/sv-jme.2016.3482

[12] Ternik, P.; Rudolf, R. (2013). Laminar natural convection of non-Newtonian nanofluids in a square enclosure with differentially heated side walls, International Journal of Simulation Modelling, Vol. 12, No. 1, 5-16, doi:10.2507/IJSIMM12(1)1.215

[13] Yuan, L.-W.; Li, S.-M.; Peng, B.; Chen, Y.-M. (2015). Study on failure process of tailing dams based on particle flow theories, International Journal of Simulation Modelling, Vol. 14, No. 4, 658-668, doi:10.2507/IJSIMM14(4)8.322

[14] FEMM 4.2 - Finite Element Method Magnetics, from http://www.femm.info/wiki/HomePage, accessed on 21-08-2016 